Revue des études hébraïques et juives

$18 \mid 2013$

Le Proche-Orient ancien à la lumière des sciences sociales

\title{
Fernand Braudel, Les Mémoires de la Méditerranée : une histoire à suivre
}

Fernand Braudel, Memory and the Mediterranean: A History to Follow

$$
\text { על ספרו של פרננד ברודל "זכרונות הים התיכון" }
$$

\section{Renée Koch Piettre}

\section{(2penEdition}

\section{Journals}

Édition électronique

URL : https://journals.openedition.org/yod/1798

DOI : 10.4000/yod. 1798

ISSN : 2261-0200

Éditeur

INALCO

Édition imprimée

Date de publication : 1 septembre 2013

ISBN : 978-2-85831-207-8

ISSN : 0338-9316

Référence électronique

Renée Koch Piettre, «Fernand Braudel, Les Mémoires de la Méditerranée : une histoire à suivre », Yod

[En ligne], 18 | 2013, mis en ligne le 01 juillet 2013, consulté le 08 juillet 2021. URL : http://

journals.openedition.org/yod/1798; DOI : https://doi.org/10.4000/yod.1798

Ce document a été généré automatiquement le 8 juillet 2021.

\section{c) (7) (8)}

Yod est mis à disposition selon les termes de la Licence Creative Commons Attribution - Pas d'Utilisation Commerciale 4.0 International. 


\title{
Fernand Braudel, Les Mémoires de la Méditerranée : une histoire à suivre
}

\author{
Fernand Braudel, Memory and the Mediterranean: A History to Follow \\ על ספרו של פרננד ברודל "זכרונות הים התיכון"
}

\author{
Renée Koch Piettre
}

1 Nous n'avons guère vocation à nous poser en évaluatrice d'un historien aussi considérable que Fernand Braudel (1902-1985), même un quart de siècle après sa disparition et même quand il s'agit d'évoquer un ouvrage portant sur un domaine du savoir qui ne relevait pas directement de sa spécialité: à savoir l'antiquité méditerranéenne. Nos propres titres en la matière ${ }^{1}$ ne sauraient pas davantage suffire à autoriser un jugement sur la vaste fresque historique dont il va être question: Les Mémoires de la Méditerranée. Préhistoire et Antiquité, une sorte d'addendum posthume (paru en 1998 seulement aux éditions de Fallois) au grand ouvrage sur La Méditerranée et le monde méditerranéen au temps de Philippe II. Braudel déclare lui-même dans un «Avertissement" n'être spécialiste que de la Méditerranée du $\mathrm{XVI}^{\mathrm{e}}$ siècle $^{2}$. Mais il devient ici préhistorien et antiquisant, brassant les ères géologiques, les millénaires, les civilisations de Gadès à Babylone, jusqu'à ce que, avec le dernier chapitre, il ait été montré comment «Rome devint la Méditerranée plus qu'entière». De discrètes annotations éditoriales corrigent déjà, à distance de sa rédaction, les données qui, depuis la rédaction de ce texte (1969), ont pu évoluer avec le temps et les progrès de l'archéologie. On peut compléter l'examen de l'ouvrage par la lecture des recueils posthumes de Fernand Braudel, dont le premier s'intitule encore Autour de la Méditerranée.

2 Le présent article n'a d'autre ambition que de proposer quelques considérations épistémologiques au sujet de l'interdépendance entre l'aire géographique méditerranéenne et les réalisations humaines qui s'y inscrivent. 


\section{Le temps rabattu sur l'espace ou la synchronie en clé épique}

3 Rappelons que Braudel a privilégié, par méthode, les grands espaces et le temps long. L'histoire événementielle ne lui paraît devoir être abordée qu'après

avoir fixé ces grands courants sous-jacents, souvent silencieux, et dont le sens ne se révèle que si l'on embrasse de longues périodes de temps. Les événements retentissants ne sont souvent que des instants, que des manifestations de ces larges destins et ne s'expliquent que par eux ${ }^{3}$.

Il y aurait donc un « sens » de l'histoire, un « destin », qui n'apparaîtrait qu'à un regard capable d'embrasser large ; une fois ce sens reconnu, les événements s'ordonneraient comme d'eux-mêmes vers leur fin. Et les grands courants où le sens s'inscrit requerraient à leur tour de larges espaces où puissent fluctuer les fleuves naturels et humains, espaces qui demandent à être au préalable reconnus, cartographiés, sondés jusque dans leurs strates géologiques. L'enquête historique, pour Fernand Braudel, va ainsi de pair avec une enquête géographique et cette enquête était en quelque sorte déjà faite dans sa thèse sur La Méditerranée au temps de Philippe II : l'espace est moins un cadre qu'un partenaire décisif, et surtout premier, à la fois originel et permanent, de l'aventure historique.

De l'observatoire des civilisations, la vue porte, doit porter très au loin, au bout de la nuit de l'histoire, et même au-delà. Faut-il avouer qu'un historien du Xvi siècle considère que la nouvelle revue de protohistoire, Chthonia-préoccupée, entre autres tâches, par l'étude des lointains substrats méditerranéens alpins et nordiques, et attentive à signaler d'archaïques résurgences dans le culte des morts - intéresse son époque ? La civilisation c'est aussi un lointain, un très lointain passé obstiné à vivre, à s'imposer et qui compte pour l'habitat et les pratiques agraires des hommes autant que le relief, le sol en place, le ravitaillement en eau ou le climat $[. . .]^{4}$

On peut élargir encore la perspective et rappeler que Fernand Braudel, qui fonda la revue des Annales et dirigea la sixième Section de l'École Pratique des Hautes Études (EPHE) pour la conduire jusqu'à son autonomie sous le nom d'École des Hautes Études en Sciences Sociales (EHESS), souhaitait voir se développer un langage commun dans toutes les sciences sociales sur la base théorique du postulat de «la réduction nécessaire de toute réalité sociale à l'espace qu'elle occupe ». Manière éminemment positiviste de rabattre le temps, d'essence toujours abstraite, sur l'espace, qui s'offre à l'étude objective. L'histoire de la Méditerranée s'identifierait ainsi au bassin même de la Méditerranée. Paradoxalement, il n'y aurait en quelque sorte d'histoire que synchronique : là où s'installe la durée, il devient possible de parler de civilisation.

Une civilisation, c'est tout d'abord un espace, une «aire culturelle » [...] Si à la cohérence dans l'espace s'ajoute une permanence dans le temps, j'appelle civilisation ou culture l'ensemble, le «total » du répertoire ${ }^{5}$.

6 Pour employer une autre image, on peut dire aussi que le personnage principal des Mémoires de la Méditerranée est le même que celui de La Méditerranée au temps de Philippe II, à savoir la Méditerranée elle-même. Deux sous-titres au moins le disent expressément : «Le vrai personnage : la Méditerranée du Levant » (p. 147); «Le grand personnage : la civilisation méditerranéenne » (p.485). L'enquête objective s'énonce avec le souffle de l'épopée. La Méditerranée est presque un personnage de mythologie, ce reste, écrit Braudel, de la primitive Téthys que Rome aurait en quelque sorte, in fine, 
fait advenir à sa propre identité, à sa propre unité, en en rassemblant toutes les parties sous sa juridiction: là où, en sens inverse, de très anciennes cicatrices et lignes de fractures, tout au long de l'histoire, se sont obstinées à se rouvrir.

\section{Une fracture méditerranéenne}

7 La Méditerranée n'aura jamais surmonté entièrement, par exemple, la fracture entre son versant sémitique et punique d'une part, indo-européen d'autre part :

Lorsque E.-F. Gautier, contre les spécialistes, soutient qu'en Afrique du Nord et en Espagne, l'Islam a retrouvé les bases puniques anciennes et que cette première civilisation a préparé le terrain à la poussée musulmane - il reste, à mon sens, dans les limites autorisées de l'hypothèse ${ }^{6}$.

8 C'est un état de choses que Braudel regrette : on croit savoir que, reçu à l'agrégation d'histoire à l'âge de 21 ans et d'abord nommé professeur en Algérie de 1924 à 1932, il montra peu d'intérêt pour la culture indigène, évaluant positivement au contraire l'implantation européenne et la colonisation ${ }^{7}$. Il se conformait en cela aux esprits de son temps. Le fait est que Les Mémoires de la Méditerranée soulignent à plus d'une occasion la fracture méditerranéenne, exprimant, un peu plus qu'à mi-mot, une préférence de l'auteur fondée sur la longue tradition historiographique de l'opposition entre Orient et Occident et de la méfiance à l'égard de l'apport oriental ${ }^{8}$. Ainsi p. 282 et suivante :

La conquête perse achevée, à la fin $\mathrm{du}_{\mathrm{VI}}^{\mathrm{e}}$ siècle, il y a une telle accumulation de puissance en Orient que le monde méditerranéen brusquement penche à nouveau vers lui. C'est par ce mouvement de bascule que le destin grec sera rejeté vers l'est pour le plus grand regret [sic] de l'auteur de ce livre !

9 L'Occident, écrit-il immédiatement après, «est devenu [dès ce moment même] une grande force d'histoire, un ouvrier du destin même de la Méditerranée », avec de larges mouvements de peuples poussés vers l'extrémité de l'«entonnoir» européen, un entonnoir à plusieurs endroits percé vers le Sud et que viendra remplir en temps et lieu "le rôle de réservoir tenu par une prodigieuse Europe centrale, sauvage encore, avec d'immenses forêts...» (p.285). Et plus haut, juste avant de se tourner vers un paragraphe intitulé « Dans les déserts du Sud : les Sémites », Fernand Braudel s'attarde un instant sur les Hittites, «le premier peuple indo-européen, écrit-il, qui nous soit connu du dedans, grâce à la documentation retrouvée à Bogazköy », et se laisse aller à rêver :

Est-ce un tort que d'imaginer un peuple honnête, courageux, les pieds bien sur terre, gai, amoureux de danse et de musique, tendre à l'égard des animaux et des enfants? De charmantes sculptures montrent le jeune prince jouant debout sur les genoux de la reine ou venant lui présenter ses exercices d'écriture. Un peuple naïf encore qui se chauffe au soleil des civilisations proches et, peu à peu, fabrique ses conventions impériales. Mais le roi hittite ne jouera jamais le dieu vivant comme le pharaon. Souverain d'un peuple de guerriers, il est de ceux cependant qui choisirent plus souvent les voies de la diplomatie que celles de la guerre pour atteindre leurs fins et l'on a remarqué, chez les Hittites, l'absence de la cruauté guerrière qui marque toute l'époque, même l'Égypte, et qui se fera terrifiante, plus tard, chez les Assyriens. Un dernier trait, significatif : le statut social des femmes on l'attendait peu de ce peuple de soldats - semble aussi libéral que celui de la Crète' . 

d'enfants en masse au tophet de Salammbo ${ }^{10} . .$. Mais l'auteur pleure, autant que le fit Scipion Émilien, sur la terrible destruction de la grande Carthage ${ }^{11}$.

11 Un rôle extraordinaire de diffuseur et de distributeur de la civilisation est accordé à la Crète comme à un centre rayonnant qui, tourné vers l'Orient et le Sud (l'Égypte), n'en a pas moins permis de recevoir aussi d'Europe ses hommes et une part de sa vitalité (comme le suggère l'écriture mycénienne dite linéaire $B$, qui dériverait de l'écriture minoenne dite linéaire $\mathrm{A}$, et qui se trouve noter du grec, c'est-à-dire une langue relevant des parlers indo-européens) : pour la période précédant ce que Braudel appelle encore le "miracle grec $~$, l'extraordinaire période faste du second millénaire avant Jésus-Christ, à l'âge du bronze, est brossée avec vigueur (non sans un accent sur l'« universalisme» amarnien, ce bref épisode dont l'interprétation suscite de si vifs débats parmi les historiens ${ }^{13}$ ), de même que le choc de sa disparition vers 1200 (les «Peuples de la mer ») et de l'entrée dans les «âges obscurs » qui signe les débuts de l'âge du fer... Il y a dans tout cela quelque chose de trop vigoureux pour demeurer dans l'exactitude et comme le résultat d'une longue décantation de la mémoire de l'historien. N'avait-il pas déjà composé sa grande thèse sur La Méditerranée au temps de Philippe II à l'écart des bibliothèques, du fond de sa captivité en Allemagne? Braudel croit aux identités construites par le temps long, comme il crut aussi à l'« identité de la France ».

12 C'est pourtant, au-delà de l'École des Annales tout entière, à de nouvelles avancées méthodologiques que, tout dernièrement, a pu mener le travail de Braudel: cette ouverture méthodologique est de portée bien plus large que les seules études du Proche-Orient privilégiées dans le présent volume; mais elle est telle, que ces lignes de fracture méditerranéennes héritées d'une vision historiographique à laquelle Braudel restait attaché (ainsi la thèse d'Henri Pirenne, dont Braudel se sentait tributaire, décrivait une Méditerranée définitivement divisée entre deux civilisations hostiles, Islam et Chrétienté) ${ }^{14}$, de même qu'une épistémologie fondée sur l'ordre du temps et la succession linéaire des causes et des effets qu'il a, cette fois, résolument dépassée, devraient pouvoir être rangées parmi les curiosités du passé.

\section{Deux prolongements de la méthode : systèmes et paysages}

13 Écartons d'abord l'historiographie des « lieux de mémoire » que nous serions tentés de rapprocher du titre Les Mémoires de la Méditerranée : la synthèse de Braudel n'a rien d'un pèlerinage vers des lieux-sources ni d'un effort de préservation muséale (pour lequel il aurait pu prendre modèle sur la Périégèse du Grec Pausanias attaché à présenter à son lecteur «toutes les choses grecques", panta ta hellênika) ${ }^{15}$. Les " Mémoires » sont chez lui strates sensibles ou semences du présent.

\section{« Network Approach »}

Prenons plutôt la méthode de l'analyse des réseaux, telle qu'elle est présentée et appliquée à l'Antiquité méditerranéenne dans l'introduction de l'ouvrage Greek and Roman Networks in the Mediterranean, paru en $2009^{16}$. 
L'ouvrage est introduit par une référence appuyée à Braudel ainsi qu'au patient travail, contemporain de celui de Braudel, de Shlomo Dov Goitein, A Mediterranean Society: The Jewish Communities of the Arab World as Portrayed in the Documents of the Cairo Geniza ${ }^{17}$. Les deux ouvrages sont présentés comme offrant les lignes directrices d'une historiographie de network approach: les networks sont en l'occurrence des réseaux formés de connexions aléatoires entre des nœuds. Ainsi un jeu internet créé par une poignée d'étudiants, les "six degrés de séparation de Kevin Bacon", prouve que n'importe quel acteur d'Hollywood peut être mis en relation avec le dénommé Kevin Bacon par l'intermédiaire d'un maximum de six autres personnes. Si un affinement de la méthode (ou peut-être la résistance des schémas anciens) a permis de distinguer des nœuds localement, relativement ou globalement centraux, et d'autres comme des points périphériques, le concept exploite aussi la notion de "rhizome » élaborée par Deleuze et Guattari et récuse les hiérarchies traditionnelles entre centre et périphérie ou le modèle de l'arbre, avec racines, tronc et branches, qu'illustrent les stemmata. Il est utilisé en sociologie (les " réseaux sociaux»), mis en pratique dans l'organisation de la recherche ou le domaine des affaires, mais demandait encore à être appliqué à l'histoire, ce qui advint notamment avec l'ouvrage de Peregrine Horden et Nicholas Purcell, The Corrupting Sea. A Study of Mediterranean History ${ }^{18}$. Une dimension dynamique et aléatoire réinsère ainsi le facteur temporel dans ce qui, chez Braudel, risquait de s'éterniser dans l'à-plat de la carte, se coaguler dans la synchronie.

\section{Histoire du paysage}

D'autre part, la géohistoire de Fernand Braudel trouve aujourd'hui une large application dans l'archéologie, dans l'histoire du paysage ou dans l'archéogéographie ${ }^{19}$ :

La reconstitution des spatiotemporalités et leur organisation en unités écouménales d'un type nouveau, passent par un examen attentif des modes d'articulation de plusieurs plans complexes, touchant aussi bien aux matérialités elles-mêmes ${ }^{20}$.

Il s'agit, explique Bruno Latour ${ }^{21}$, d'établir de nouvelles connexions « entre le passé et le présent, entre le local et le global, entre les natures et les cultures ». Ce qui suppose une cascade de remise en cause, "jusqu'à des propos théoriques sur l'aventure de la modernité en passant par une critique, souvent impitoyable, de l'épistémologie des disciplines canoniques ». "Ne plus utiliser comme véhicule de transport dans le temps, les explications fournies par les disciplines», pratiquer une sorte de "cure d'amaigrissement explicatif ", à partir notamment d'une "critique systématique des catégories a priori de temps et d'espace»: tout se passe, continue Bruno Latour, " comme si le temps et l'espace se morcelaient et se courbaient autour des singularités repérées par l'enquête archéologique ou géographique ». Au lieu des grands espaces méditerranéens, la méthode se concentre ainsi sur des "unités » minimales qui recèlent pourtant et travaillent les traces du tout. Il s'agit aussi de ne pas opposer tout uniment les données naturelles et les données sociales: Philippe Descola a montré que nature et culture sont coproduites, ensemble, et l'une par l'autre ${ }^{22}$. On s'appuiera, de ce fait, sur ces sciences hybrides que sont la géographie et l'archéologie. On remplacera les cartes planes par des graphes intégrant la dimension temporelle. Et l'on participera ainsi (à partir de l'étude d'objets concrets, en plaçant en quelque sorte la tentation de la théorisation en attente, en la suspendant provisoirement) à actualiser la notion de territoire à l'ère de la troisième globalisation (l'âge de la Terre) ${ }^{23}$, à remettre en cause la manipulation des faits du passé et la notion de patrimoine exploitée à des fins 
nationalistes, etc. Un exemple éclairant de la fécondité de la méthode est l'étude de la centuriation romaine en tant qu'elle a profondément modelé le paysage français ${ }^{24}$. On trouverait les prémisses d'une telle approche dans le Tableau de la géographie de la France de Paul Vidal de La Blache que Braudel n'ignorait pas (du même auteur, La Péninsule européenne. L'Océan et la Méditerranée, préfigurent davantage les vastes espaces embrassés par Braudel dans Les Mémoires de la Méditerranée) ${ }^{25}$.

Une approche plus molle, si l'on peut dire, du paysage passe par le renoncement au vieux schéma de l'opposition entre sujet et objet, regardant et regardé, dont la théorisation la plus puissante, encore largement ignorée du monde scientifique, est celle de Niklas Luhmann ${ }^{26}$. Il y a, entre l'acteur historique et le paysage, un rapport d'immanence réciproque. Comme l'écrit W.J.T. Mitchell, tout paysage peut être perçu à la fois comme "signifiant et signifié, cadre et ce qui est encadré, réalité et représentation de la réalité, récipient et contenu du récipient ${ }^{27}$ : il énonce, écrit P. N. Doukelis ${ }^{28}$, « des réalités, des situations, des aspirations beaucoup plus complexes que l'entité géographique et spatiale à laquelle il correspond. En ce sens, il n'est pas de paysage neutre, de ce qu'il était convenu d'appeler un paysage en forme de toile de fond. Bien plutôt, un paysage est un creuset d'informations [...] où se manifestent rapports et hiérarchies sociales [...] » Cette interdépendance, si elle ne s'attardait pas sur le détail des paysages, est évidente déjà chez Braudel.

\section{Les détails de la grande Histoire}

19 On peut comprendre que, dans l'histoire de la Méditerranée ancienne si largement brossée par Fernand Braudel sur un substrat géographique et paléographique, à partir de données matérielles plus qu'à partir des documents textuels, avec une attention au quotidien plus qu'à l'événement, certains phénomènes purement locaux soient passés quasi sous silence. Que des phénomènes (épiphénomènes ?) littéraires ou religieux, face à l'abondance des traces de la civilisation matérielle, soient ignorés dans les analyses. Ainsi en est-il des Hébreux et du judaïsme et ce quasi-silence (au moins par rapport à la profusion des études afférentes ou au caractère conventionnel de la place qui leur est faite parmi les disciplines universitaires encore tributaires d'un héritage théologique) a quelque chose d'intéressant en soi. Ainsi Les Mémoires de la Méditerranée signalent, à la fin d'un paragraphe sur les Araméens, qu'« au milieu de ces complications les Hébreux s'installèrent (avant 1230 semble-t-il) dans les montagnes à demi-vides de la Palestine, les Cananéens et les Philistins leur interdisant l'accès des plaines. Dernière vague, en arrière-garde, les Arabes sont signalés au IX ${ }^{e}$ siècle, pour la première fois, par les textes babyloniens. Mais la grande histoire attendra longtemps leurs hauts faits » (p. 255). Une page (275-276) est consacrée à l'État juif du temps de Salomon, sous l'enseigne d'une sorte de «balkanisation » du Proche-Orient, mais les Juifs, dit Braudel, comme les Grecs et les Hittites, «s'enlisent dans le bien d'autrui » et ce sont des Phéniciens de Tyr qui construisent le temple et le palais de Jérusalem au temps de Salomon ou font pour le roi juif le voyage d'Ophir par le long chemin de la mer Rouge : «nul n'aurait pu prévoir alors, conclut l'auteur, les malheurs proches et le rôle fabuleux que l'avenir réservait au message spirituel d'Israël qui mûrit lentement, au milieu des intempéries de l'Histoire ». Ailleurs encore, les possessions de Salomon sont décrites comme des "terres médiocres, souvent aux mains de pauvres populations semi-nomades" (p.308). Des «guerres des Juifs» nous apprenons seulement, au sujet du règne de 
Trajan, que "les Juifs et les Arabes [étaient] violemment irréductibles» (p. 483). Et, pour finir, c'est le déplacement à Constantinople de la capitale romaine qui semble l'événement essentiel capable de donner corps à l'«irruption du christianisme", lequel, ainsi indiqué en sous-titre, est pourtant recouvert, dans les courts paragraphes qui suivent, par les développements nouveaux de la philosophie grecque, la théurgie, les cultes orientaux, les thaumaturges comme Apollonios de Tyane, et les «marchands d'Orient, ces Syri que l'on retrouve partout, Juifs ou Syriens » (p. 512).

Un paragraphe de Braudel s'intitule «la revanche de Spartacus» (p.436): de quoi s'agit-il ? Du fait que l'abondance de la main-d'œuvre servile a ôté aux Grecs et aux Romains la possibilité de tirer parti de la science et des techniques alexandrines. Curieusement, Braudel, qui dans le cas des Hébreux s'est explicitement abstenu dans notre ouvrage de toute projection vers l'avenir qui attendait ce peuple, le rivant strictement à son insignifiance géographique ou politique dans l'époque considérée, anticipe ici l'avenir comme si le développement technique de l'Occident avait déjà été contenu dans l'ingénierie hellénistique, comme une nécessité freinée par un simple aléa: la civilisation matérielle relèverait de contraintes prévisibles que la culture intellectuelle ou religieuse ignore. Donnons un exemple récent d'une démarche radicalement opposée : une démarche qui tire de la coïncidence entre deux événements périphériques, d'une notule d'historien, d'un fragment de papyrus des conséquences significatives, voire de grandes conséquences. Je ne prétends pas que la méthode soit la plus recommandable (son application, bardée de bonnes intentions, pèche trop souvent par un défaut de rigueur), mais elle n'est pas sans bénéfice : comparons l'évocation de Spartacus par Braudel avec un chapitre de l'ouvrage de Page duBois intitulé Out of Athens. The New Ancient Greeks ${ }^{29}$. Ce chapitre porte sur Spartacus: Page duBois insiste, elle, sur la coïncidence qui fit de cet esclave thrace assisté selon Plutarque d'une femme prophétesse de Dionysos, la victime du général romain Crassus; Crassus fit crucifier tous les survivants de la rébellion ; il mourut lui-même, à l'autre bout de l'empire, sous les coups des Parthes, et sa tête se trouva brandie, devant le roi d'Arménie en train de banqueter, par un acteur interprétant le rôle d'Agavé en proie à la possession dionysiaque et brandissant la tête de son propre fils dans les Bacchantes d'Euripide. Un autre chapitre du même ouvrage est consacré aux supplices des esclaves, parmi lesquels des Juifs, dont Jésus. Il nous semble que l'événement périphérique vaut quelquefois d'être mis en exergue. Il peut remettre en cause tout un tableau d'ensemble. Ce n'est pas qu'il faille nécessairement prôner une histoire en haillons, remplacer la grande histoire événementielle non, cette fois, par l'histoire des civilisations dans leur dimension matérielle et factuelle, mais par l'histoire anecdotique révélatrice du continent des exclus: il reste vrai cependant que des événements minuscules peuvent effectivement renverser le sens de l'histoire et mêler l'Occident à l'Orient, la Grèce, Rome et les Sémites par exemple, d'une manière a posteriori signifiante.

\section{Un prédécesseur : I'historien Polybe et l'avènement de réseaux entre Orient et Occident méditerranéens}

21 Je voudrais ici faire intervenir le grand prédécesseur de Braudel dans sa représentation de l'histoire méditerranéenne comme un tout organique : à savoir Polybe. Mais Polybe s'intéresse, lui, aux guerres, aux hommes, aux événements. De ce fait il peut arriver 
chez Polybe que des connexions de détail acquièrent une grande importance dans l'histoire globale de la Méditerranée de son temps et ce sont toutes ces connexions qu'il repère et met en évidence. Évoquons nommément, dans les Histoires de l'Achéen, un épisode des relations entre Rome et le royaume séleucide. On sait comment le roi séleucide Antiochos IV, de retour en Syrie après sa longue captivité dorée à Rome, n'eut de cesse de reproduire en son royaume oriental tout ce qu'il avait pu admirer dans la Ville aux sept collines. Ainsi il se promenait en toge, jugeait assis sur un siège curule et jouait à briguer des voix à la façon romaine ${ }^{30}$. Zeus devenait un autre Jupiter Capitolin à qui il fit élever des temples magnifiques, dont celui, qu'il voulut fastueusement compléter et agrandir, de Zeus Olympien à Athènes... Polybe, proche des cercles du pouvoir romain, nous révèle que lui-même veilla en personne à organiser en 162 av.n.è. l'évasion de l'otage syrien qui avait remplacé Antiochos à Rome, à savoir Démétrios (le futur Démétrios ${ }^{\text {er }}$ ), neveu d'Antiochos et fils de Séleucos IV. L'historien nous raconte à cette occasion l'assassinat, dans un gymnase de Laodicée-sur-Mer (l'actuelle Lattaquié en Syrie), de l'ambassadeur romain $\mathrm{Cn}$. Octavius et il observe en cette circonstance deux caractères assez curieux : deux hommes de Laodicée, l'assassin d'une part, nommé Leptinès, qui était si sûr de son bon droit et si sûr que Rome saurait lui donner raison qu'il voulut même s'expliquer devant le Sénat; et un autre personnage, un grammairien nommé Isocratès, qui donnait des conférences publiques, disait haut et fort « que Cn. Octavius n'avait eu que ce qu'il méritait, qu'il fallait tuer aussi les autres commissaires romains pour que personne ne pût apporter la nouvelle à Rome et qu'ainsi on en finirait avec les injonctions arrogantes et la tyrannie effrénée des Romains ", mais se laissa complètement aller, jusqu'à paraître un véritable monstre humain, dès qu'il fut mis aux fers. C'est précisément pour avoir appris ce grave incident diplomatique que Démétrios, alors otage à Rome, avait pris le parti de s'évader et la première chose qu'il fit ensuite, ce fut de demander la caution de Rome avant de ceindre le diadème, puis d'envoyer au Sénat, en guise de gage, le meurtrier d'Octavius. Mais le Sénat dédaigna ces gages trop faciles ${ }^{31}$.

Ces faits ont-ils quelque chose à voir avec la civilisation méditerranéenne, ou bien ne s'agit-il que d'événements politiques sans autre incidence sur le destin de la Méditerranée? Nous avancerons ici notre propre interprétation. Nous suggérons que ce qui peut nous intéresser dans cette histoire, au-delà de ces allers-retours entre Rome et Antioche, c'est que le meurtre ait eu lieu dans un gymnase et cela, à Laodicée-surMer. Un petit jeune homme, semble-t-il, croit agir pour le bien en tuant un tyran venu brûler la flotte militaire et couper les jarrets des éléphants pour briser la puissance militaire séleucide. Il s'imagine même pouvoir se faire entendre du Sénat. Rome, dont il avait tué le représentant, restait malgré tout à ses yeux un havre de justice et de sûreté. Il était sûr de son bon droit. Qu'est-ce qui lui donnait cette assurance? Son éducation peut-être?

Qu'enseignait-on au gymnase de Laodicée-sur-Mer?

Eh bien, peut-être qu'on y enseignait la doctrine d'Épicure. En effet, le grand homme de Laodicée en ce temps-là s'appelait Philonidès. C'était un philosophe et mathématicien épicurien, mais aussi un personnage reconnu, à l'occasion chargé de missions diplomatiques et accueilli à la cour syrienne, peut-être en qualité d'historien officiel ${ }^{32}$ depuis le règne de Séleucos IV (187-175), si l'on en croit une relecture par Dov Gera ${ }^{33} \mathrm{du}$ papyrus, très fragmentaire, qui nous a conservé sa Vita ${ }^{34}$. Il semble qu'il s'entremit en faveur de sa cité, pour lui éviter une punition collective ${ }^{35}$, en une occasion que Dov Gera 
place en 175, au moment où Séleucos IV venait d'être assassiné (à Laodicée même ?) et où son ministre Héliodore accaparait par là même le pouvoir qui avait été brièvement confié à un enfant nommé Antiochos, le tout jeune fils de Séleucos IV, avant que n'intervienne son oncle, le futur Antiochos IV: celui-ci se débarrassa d'Héliodore, épousa la veuve de son frère et prit l'enfant sous sa tutelle, cependant que le second fils de Séleucos IV, le futur Démétrios I ${ }^{\text {er }}$, était envoyé à Rome comme otage. Crönert, le premier éditeur de la Vie de Philonidès (1900) et Italo Gallo son second éditeur avaient supposé, de leur côté, que l'occasion de ce service extraordinaire rendu à sa cité avait été justement l'assassinat de $\mathrm{Cn}$. Octavius en 162 sous l'interrègne suivant, après la mort d'Antiochos IV et sous le bref règne de son fils Antiochos V. Mais tout laisse croire que Philonidès a pu en effet secourir sa patrie lors du premier interrègne, grâce à sa position déjà acquise à la cour où un précepteur des jeunes princes semblait lui-même " ne pas être opposé à la doctrine ${ }^{36}$ et donc avoir pu sensibiliser ses élèves à la cause de la patrie de Philonidès. Il s'ensuivrait que le futur Démétrios IV, lorsqu'il séjourna à Rome, aurait déjà été imbu des rudiments d'une philosophie épicurienne dont, à son retour en Syrie, il se montra l'ardent émule, offrant à Philonidès une maison à la porte de son palais et se rendant assidûment à ses leçons ${ }^{37}$. Le geste de Leptinès en plein gymnase de Laodicée-sur-Mer pourrait dans ce cas avoir profité en 162 d'une impunité qui se serait antérieurement déjà manifestée, sans doute avec de solides arguments de la part de Philonidès... La brève crise diplomatique entre Rome et la Syrie n'illustrerait pas seulement la mise en place d'un réseau d'échanges entre les deux parties de la Méditerranée, il n'y aurait pas là un simple détail dans le processus d'unification du bassin méditerranéen. Car il ne serait pas indifférent qu'Antiochos IV, le souverain oriental imbu d'usages romains, celui qui par ailleurs prétendit réformer le culte du dieu de Jérusalem à la mode du Zeus Olympien d'Athènes ou du Jupiter Capitolin de Rome, précipitant chez son voisin juif une hellénisation forcée et déclenchant ainsi la révolte des Maccabées, ait pu être courtisé par la première secte philosophique à avoir sérieusement prétendu aligner la représentation des dieux sur un dogme tenu pour seul véridique ${ }^{38}$. L'événement d'allure périphérique que nous venons de pointer deviendrait alors un symptôme et un déclencheur d'une crise religieuse qui bientôt intéressera la Méditerranée entière.

\section{Conclusion}

Pour conclure en un mot, nous dirons qu'au-delà de la brillante synthèse que constituent Les Mémoires de la Méditerranée et de son efficacité pédagogique comme de l'agrément de sa lecture, il faut reconnaître dans le regard de Braudel sur la Méditerranée antique une incitation à la mise en œuvre de la collaboration aujourd'hui nécessaire des sciences de la nature avec les sciences humaines et non plus des seules sciences humaines dans leur ensemble. Les grandes lignes qu'il traça restent comme une carte approximative à très grande échelle qui malgré ses inexactitudes sert encore de boussole tant aux apprentis historiens qu'aux chercheurs courbés sur leur terrain archéologique ou cheminant au ras de sinuosités et de remous créés par des événements minuscules. Car il resterait aussi à rendre toute sa place, dans l'Histoire, au rôle des événements et des idées. 


\section{NOTES}

1. Avec nos collègues Francis Schmidt et Stella Georgoudi et en qualité d'helléniste, nous avons coanimé entre 1999 et 2006 un séminaire interdisciplinaire sur les religions et les sociétés de la Méditerranée ancienne (EPHE et ex-Centre Gustave Glotz de Recherches sur les mondes hellénistique et romain). De ce séminaire et de deux tables rondes organisées à sa suite, nous avons tiré deux ouvrages collectifs, La cuisine et l'autel. Les sacrifices en questions dans les sociétés de la Méditerranée ancienne, Turnhout, Brepols, 2005 (Bibliothèque de l'École des Hautes Études, Sciences religieuses 124) et La Raison des signes. Présages, rite, destin dans les sociétés de la Méditerranée ancienne, Leiden, Brill, 2012 (Religions in the Graeco-Roman World 174).

2. «Tout compte fait, mes recherches personnelles ne couvrent vraiment que la période 1450-1650 », p. 21 de l'édition en Livre de poche (2001) à laquelle nous nous référons tout au long du présent article (Fernand Braudel, Les Mémoires de la Méditerranée, Paris, Éditions de Fallois, 1998).

3. Fernand Braudel, Écrits sur l'histoire, Paris, Flammarion, 1985, p. 13.

4. Braudel, La Méditerranée et le monde méditerranéen au temps de Philippe II, Paris, Armand Colin, $2^{\mathrm{e}}$ éd. rév. 1966 [1 ${ }^{\text {re }}$ éd. 1949], II, p. 505.

5. Ibid., p. 292.

6. Ibid., p. 504.

7. Voir Claude Liauzu, «La Méditerranée selon Fernand Braudel », Confluences Méditerranée, 31, 1999, p. 179-187 : «Au fond, la Méditerranée selon Fernand Braudel est un lac occidental où l'islam est un intrus » (p. 186).

8. Renvoyons notamment à Edward W. Said, L'Orientalisme. L'Orient créé par l'Occident [1978], trad. fr. de Catherine Malamoud [19801ㅣ, Paris, Seuil, 2003.

9. Braudel, Les Mémoires de la Méditerranée, p. 250-251.

10. On discute toujours du crédit à accorder à des auteurs comme, par exemple, Diodore de Sicile, Xx, 14, 6; Denys d'Halicarnasse, Antiquités de Rome, I, 38, 2 ; Porphyre, De l'abstinence, II, 56, 1, citant « Sanchuniathon » d'après Philon de Byblos ; Plutarque, De la Superstition, 13 (Moralia 171 BC) ; Tertullien, Apologétique, IX, 2-3... bientôt relayés par la tradition rabbinique.

11. Cf. Polybe xxxvIII, 21-22 (xxxIX, 5, 1-3 et 6, 1-3), d'après Appien 132.

12. Qui sera contesté par Louis Gernet, Jean-Pierre Vernant ou Riccardo di Donato : voir Riccardo di Donato (éd.), Les Grecs sans miracle, préface de Jean-Pierre Vernant, Paris, La Découverte/ Maspero, 1983.

13. Braudel se situe en deçà de la problématique développée par Jan Assmann, Moïse l'Égyptien. Un essai d'histoire de la mémoire, traduit de l'allemand par L. Bernardi, Paris, Aubier, 2001, et le débat suscité par l'ouvrage. Voir aussi Jacques Le Rider, "Moïse égyptien», Revue Germanique Internationale, 14, 2000, p.127-150 [en ligne, URL: http://rgi.revues.org/811, consulté le 16 janvier 2011].

14. Henri Pirenne, Mahomet et Charlemagne, Paris, PUF, coll. "Quadrige/Grands textes ", 2005 [1937] : c'est l'invasion de l'islam qui aurait mis fin au Moyen Âge. Thèse élaborée en captivité pendant la Première Guerre mondiale, comme le fut la thèse de Braudel au cours de la Deuxième Guerre mondiale. Sur la «bibliothèque » de Braudel, voir Érato Paris, La Genèse intellectuelle de l'œuvre de Fernand Braudel, Paris, éd. Fides, 2002 ; également Claude Liauzu, op. cit., p. 184.

15. Voir Vinciane Pirenne, Retour à la source, Pausanias et la religion grecque, Liège, 2008.

16. Irad Malkin, Christy Constantakopoulou, Katarina Panagopoulou (éds), Greek and Roman Networks in the Mediterranean, London \& New York, Routledge, 2009 : «Introduction », p. 1-11. 
17. Shlomo Dov Goitein, A Mediterranean Society: The Jewish Communities of the Arab World as Portrayed in the Documents of the Cairo Geniza, 5 vol. , Berkeley, Los Angeles, London, Berkeley U.P., 1999.

18. Peregrine Horden, Nicholas Purcell, The Corrupting Sea. A Study of Mediterranean History, Oxford, Blackwell, 2000.

19. Voir par exemple Gérard Chouquer, Quels scénarios pour l'histoire du paysage? Orientations de recherche pour l'archéogéographie, Coimbra, Porto, 2007 ; ou encore Philippe Leveau, « Archéologie, espace et environnement: des paysages aux risques naturels» in François Dumasy, François Queyrel (éds), Archéologie et environnement dans la Méditerranée antique, Genève, Droz, 2009, p. 1-22; et les actes d'une rencontre à Santorin : Panagiotis N. Doukelis (éd.), Histoires du paysage, Athènes, 2007.

20. Chouquer, op. cit., p. 184.

21. En guise de préface à l'ouvrage de G. Chouquer.

22. Philippe Descola, Par-delà nature et culture, Paris, Gallimard, 2005.

23. Après la «deuxième globalisation" (découpage des nations et des frontières) et la « première » (le globe et la cartographie).

24. La centuriation romaine est le schéma géométrique du plan d'une ville et du territoire agricole environnant utilisé dans le monde romain : il était tracé dans chaque nouvelle colonie à l'aide des instruments d'arpenteurs.

25. Paul Vidal de La Blache, La Péninsule européenne. L'Océan et la Méditerranée, Paris-Nancy, 1873 ; Tableau de la géographie de la France, Paris, Hachette, 1903. Voir Larissa Alves de Lira, "Fernand Braudel e Vidal de La Blache: Geohistoria e Historia da Geografia », Confins [en ligne], 2, 2008, URL : http://confins.revues.org/2592, consulté le 18 janvier 2012.

26. Pour mesurer l'impact de cette théorie des systèmes sociaux, voir Hugues Rabault, «L'apport épistémologique de la pensée de Niklas Luhmann: un crépuscule pour l'Aufklärung?", Droit et Société, 42-43, 1999. En ligne, http://www.reds.msh-paris.fr/publications/revue/biblio/ds042043b.htm, consulté le 16 janvier 2012.

27. W. J. T. Mitchell, Landscape and Power: Space, Place and Landscape, Chicago, U of Chigago P, 2002 [1994].

28. Panagiotis N. Doukelis (éd.), Histoires du paysage, Athènes, 2007, p. 11. Voir aussi le numéro 4, 2010 de la Revue de l'histoire des religions, présenté par John Scheid et François de Polignac.

29. Page DuBois, Out of Athens. The New Ancient Greeks, Cambridge, MA, Harvard U.P., 2010.

30. Polybe, Xxvi, 1.

31. Polybe. Voir aussi Cicéron, $9^{\text {e }}$ Philippique, 3 : «À Cn. Octavius, cet illustre et grand citoyen qui le premier fit entrer le consulat dans une famille depuis si féconde en grands hommes, fut érigée la statue que nous voyons encore auprès des Rostres. Personne alors ne portait envie aux hommes nouveaux, personne alors qui n'honorât la vertu. Notez que cette ambassade de Cn. Octavius ne présentait pas la plus légère apparence de danger. Envoyé par le sénat pour reconnaitre les dispositions des rois et des peuples libres, et surtout pour empêcher le petit-fils de ce roi Antiochus, qui avait fait la guerre à nos ancêtres, d'avoir des flottes et d'entretenir des éléphants, il fut, dans le gymnase de Laodicée, assassiné par un certain Leptines. Alors lui fut décernée par nos ancêtres, en compensation de la vie, une statue destinée à honorer longtemps sa race illustre, dont elle est aujourd'hui l'unique monument. »

32. Italo Gallo, Vita di Filonide epicureo (PHerc. 1044) in Id., Frammenti biografici da papiri, II, Rome, 1980, 21-166, fr. 28.

33. Dov Gera, "Philonides the Epicurean at Court: Early Connections", Zeitschrift für Papyrologie und Epigraphik, 125, 1999, pp. 77-83.

34. Au milieu de la bibliothèque de l'Épicurien Philodème préservée par les cendres du Vésuve à Herculanum.

35. Vita di Filonide epicureo, fr. 9. 
36. Mê ellotriôsthai pros tên hairesin : Vita di Filonide epicureo, fr. 32.

37. Vita di Filonide epicureo, fr. $12 ; 19 ; 27$.

38. Il s'agissait d'obtenir des eidôla, des images mentales (et, accessoirement, si possible sculptées) conformes à la doctrine et non plus, comme partout jusqu'alors, émanées de l'usage. Voir notre article «'Il faut faire les statues des dieux joyeuses et souriantes' : Diogène d'ÆEnoanda (fragment $19 \mathrm{Smith}$ ) en réformateur de la religion olympienne dans la Grèce d'époque romaine » in R. Deconinck, M. Watthee-Delmotte (éd.), L'idole dans l'imaginaire occidental, Paris, L'Harmattan, 2005, p. 95-108. Pour une première formulation de notre hypothèse concernant Antiochos IV, voir aussi les dernières pages de notre article "Philonidès de Laodicée et le canon épicurien ", Cahiers Glotz, 21, 2010, p. 385-408. Jan Assmann, Moïse l'Égyptien, op. cit., situait la « distinction mosaïque » (l'affirmation d'une différence irréductible, de la non-traductibilité d'un culte) non dans l'affirmation monothéiste, mais dans la prétention à poser un culte contre tous les autres. La doctrine épicurienne, professée par un homme de pouvoir, a pu constituer, malgré elle peut-être, une figure fondatrice d'une telle intolérance.

\section{RÉSUMÉS}

Dans Les Mémoires de la Méditerranée (publication posthume en 1998), Fernand Braudel a relu l'histoire de la Méditerranée ancienne comme une unique aventure dont le bassin méditerranéen, dans sa configuration géographique et géologique, formerait l'acteur principal. Une fracture y est cependant soulignée et regrettée, entre l'Orient et l'Occident : fracture que les événements auraient contribué à aggraver malgré des périodes fastes de réconciliation. Cette approche de l'histoire par l'espace où elle se déroule, trouve aujourd'hui des prolongements dans l'étude des réseaux (network approach) et dans l'histoire du paysage qui se concentre sur des « unités écouménales » pour saisir le global par le local. Une approche inverse, qui privilégie les exclus de la grande histoire, le détail, l'événement le plus mince, pourrait avoir quelquefois une portée à grande échelle spatio-temporelle : ainsi du meurtre, en Syrie, d'un légat romain, en l'an 162 av.n.è. Cet épisode de l'unification de la Méditerranée sous le pouvoir de Rome se place dans des chassés-croisés entre Orient et Occident qui pourraient aussi avoir été porteurs d'évolution intellectuelle, voire religieuse.

In his posthumous Memory and the Mediterranean, Braudel interprets the history of the Ancient Mediterranean as a unique adventure in which the Sea, its geographical and geological setting, is the major actor/subject. Nevertheless, one can notice, and even deplore, the historical split between East and West, enhanced and intensified by the subsequent events, in spite some periods of reconciliation. The legacy of this spatial approach of history is to be recognized in some of the contemporary historiographical trends: the network approach and the history of the landscape the study of "oecumenal units" in order to apprehend the global through the local. There are also situations in history where the marginal, the detail or the contingent event have global consequences: such event was for example the murder of the Roman legatus in Syria, in 162 B.C. Also, the unification of the Mediterranean under Rome is part of those give-and-take processes between Orient and Occident leading to intellectual and religious changes. 
INDEX

\section{מילות מפתח}

המזרח התיכון, פרננד ברודל, הסטוריה של הנוף, הסטוריה ברשת:

Keywords : Braudel Fernand (1902-1985), Mediterranean, network history, landscape history, event, Middle East

Mots-clés : Braudel Fernand (1902-1985), Méditerranée, histoire en réseaux, histoire du paysage, événement

Index géographique : Méditerranée, Proche-Orient 